OPEN ACCESS

Edited by:

Éric Laurent,

Université Bourgogne

Franche-Comté, France

Reviewed by:

Elise Dan-Glauser,

University of Lausanne, Switzerland

Gilles Chopard,

Centre Hospitalier Universitaire

de Besançon, France

${ }^{*}$ Correspondence:

Maksymilian Bielecki

mbielecki@swps.edu.pl

Specialty section:

This article was submitted to

Psychopathology,

a section of the journal

Frontiers in Psychology

Received: 03 June 2017 Accepted: 01 September 2017

Published: 19 September 2017

Citation:

Bielecki M, Popiel A, Zawadzki B and Sedek G (2017) Age As Moderator

of Emotional Stroop Task

Performance in Posttraumatic Stress

Disorder (PTSD).

Front. Psychol. 8:1614.

doi: 10.3389/fpsyg.2017.01614

\section{Age As Moderator of Emotional Stroop Task Performance in Posttraumatic Stress Disorder (PTSD)}

\author{
Maksymilian Bielecki ${ }^{*}$, Agnieszka Popiel${ }^{1}$, Bogdan Zawadzki and Grzegorz Sedek ${ }^{1}$ \\ ${ }^{1}$ Department of Psychology, SWPS University of Social Sciences and Humanities, Warsaw, Poland, ${ }^{2}$ The Robert B. Zajonc \\ Institute for Social Studies, University of Warsaw, Warsaw, Poland
}

Emotional Stroop task (EST) has been extensively used to investigate attentional processes in posttraumatic stress disorder (PTSD). Even though aging significantly changes the dynamics of emotion-cognition interactions, very little is known about its role in shaping EST performance in PTSD patients. In the present study we tested a uniquely large sample of motor vehicle accident victims. Data of 194 participants (75.3\% female; mean age $=36.64$ years, $S D=12.3$ ) were included in the analysis, out of which $136(70.1 \%)$ were diagnosed with PTSD. Prior to the psychiatric assessment, participants completed the pictorial version of EST (neutral, positive, negative, and accidents photos were presented). Comparison of the PTSD and control groups revealed a specific increase in reaction times (RTs) related to the exposure of traumarelated material. At the same time, previously unreported, moderating effects of age were also discovered. Older participants, in contrast to the younger group, showed no increase in RTs and interference scores in trials where accident photos were presented. Our study points to the key role of age as a previously understudied factor modifying EST performance in PTSD patients.

Keywords: PTSD, emotional Stroop task, pictorial emotional Stroop task, attentional bias, aging

\section{INTRODUCTION}

Emotional Stroop task (EST) is one of the most important paradigms used to investigate attentional biases in a wide range of clinical populations including patients diagnosed with posttraumatic stress disorder (PTSD; Williams et al., 1996; McLeod, 2005). In the classic version of the EST, participants are asked to name the colors of sequentially presented words ignoring their meaning. Processing of the emotional content of the words imposes a cognitive load and leads to the delayed color naming. This delay, reflected in longer reaction times (RTs), is called emotional Stroop effect (ESE). In psychopathology of anxiety and stress-related disorders longer RTs are typically observed if stimuli are associated (emotionally or semantically) with participant's perceived area of threat, and thus to a clinical diagnosis. Although some of the reported results are inconsistent (Kimble et al., 2009), current consensus clearly indicates that patients with PTSD performing the EST exhibit a specific increase in RTs when confronted with trauma-related stimuli (Cisler et al., 2011). The validity of the link between PTSD and emotional Stroop effect was further corroborated in studies showing its reduction after completing psychotherapy (Foa and Rauch, 2004) or special attentional training (Khanna et al., 2016). 
There are also significant developments in our understanding of the cognitive mechanisms and neural underpinnings responsible for the emergence of the ESE, both in healthy (Ben-David et al., 2012; Ben-Haim et al., 2014) and clinical populations, including patients with anxiety disorders (for review, see: Cisler and Koster, 2010) and, more specifically, PTSD (Bruce et al., 2013; Shvil et al., 2013). There is also a growing body of knowledge concerning factors modifying the size of the ESE depending on the specific characteristic of the experimental procedure. For example, meta-analysis performed by Phaf and Kan (2007) showed that there was no detectable ESE if stimuli were shown "suboptimally," i.e., using short presentation times and/or masking reducing the conscious perception of the presented material. ESE was also systematically larger in studies presenting blocks of homogenous stimuli when compared with experiments randomly mixing neutral and trauma-related words (see also Ben-Haim et al., 2014).

Despite the breadth of research using EST, existing data on effects of age are limited and partially contradictory. While some studies describe increase in ESE with age (Wurm et al., 2004), other suggest that older adults might be less affected by the emotional content of the stimuli - either in general (Lamonica et al., 2010) or in specific experimental settings (Ashley and Swick, 2009). Authors discussing these findings refer to many different non-exclusive explanations of the observed effects. Postulated mechanisms include age-related improvement in emotional regulation (Scheibe and Carstensen, 2010; Morgan and Scheibe, 2013), differences in amygdala responsiveness to threat signals (Lamonica et al., 2010), and age-related changes in working memory functioning (Ashley and Swick, 2009). The data concerning age effects in individuals exposed to traumatic experiences are even scanter. There are only two published papers (Wittekind et al., 2010, 2017) directly tackling that issue. Both tested a group of older adults traumatized during or just after the II World War. Obtained results were partially incoherent (no ESE in the earlier study) and hard to generalize due to the limited power, specificity of the tested sample (i.e., participants were diagnosed and tested more than 60 years after the traumatic events, the PTSD group included the subsyndromal cases), and limitations of the study design not including any younger control group.

Taking into account the multitude of potential theoretical explanations, diversity of the experimental procedures, and scarcity of the empirical material it is not yet possible to create a comprehensive theoretical model of the ESE that would allow to predict the moderating role of age in the PTSD population. At the same time, however, accurate identification of this role in clinical samples is essential for a number of reasons. Firstly, it would let to determine the limitations of the concurrent and predictive validity of the ESE when used in clinical setting. ESE is often proposed as an index that could support diagnosis in practical contexts, i.e., assessing changes induced by treatment (e.g., Gropalis et al., 2013) or predicting its success (Waters et al., 2003; Streeter et al., 2008). Secondly, it would allow to further verify the existing models of attentional biases of studied disorders. Finally, it might help explain some of the discrepancies in the literature on PTSD patients, as some publications (e.g., Kimble et al., 2009) question the very existence of the ESE in this population.

It is worth noticing that our current knowledge about PTSD does not allow us to address many other basic questions related to aging, including the fundamental one - whether or not age is significantly related to the risk of PTSD development following traumatic events. Current results provide mixed evidence (e.g., meta-analysis by Xue et al., 2015) and suggest that this relationship might be shaped by many variables, including social, economic, and cultural factors (e.g., Norris et al., 2002). Hence, looking at this issue from a broader perspective, a better understanding of the processes influencing participants' behavior in relatively simple paradigms like EST might be also helpful in understanding the mechanisms of the disorder development and maintenance across the age-span.

According to our knowledge, our study is the first ever to directly address the question of the moderating effects of age on the ESE in the typical population of trauma-exposed subjects. Uniquely large sample with age ranging from 18 up to 69 years allowed us to use regression approach that has never been applied to study aging effects in PTSD. It is also the largest ever study conducted using the EST in a group homogenous regarding the type of stressor - all tested participants were motor vehicle accident (MVA) victims. At the same time, it is the only one using pictorial EST in this group, earlier reported effects were obtained using a verbal version of the task (e.g., Devineni et al., 2004). As outlined above, the ambiguity of the existing empirical material on aging effects on the ESE and limitations of data relevant to the PTSD patients determined the exploratory nature of our work. Drawing on the existing literature and concerning the sample size, we expected to observe significant effects related to the exposure of trauma-related material. We did not, however, form specific hypotheses addressing the moderating effects of age.

\section{MATERIALS AND METHODS}

\section{Study Design and Participants}

This study was approved by institutional ethics committees at the University of Warsaw and the Military Institute of Aviation Medicine in Warsaw. It was conducted at the outpatient treatment center at the University of Warsaw during the screening phase of the program TRAKT. TRAKT (meaning "route" in Polish) was a clinical trial comparing the efficacy of different treatment modalities for PTSD (paroxetine vs. prolonged exposure vs. combined treatment) following MVA (for details: see Popiel et al., 2015).

\section{Participants}

The sample comprised of 202 participants who underwent clinical assessment at one of the diagnostic centers involved in the TRAKT project. Participants included in this sample completed a battery of computerized tests (including pictorial EST). Four participants were excluded on an a priori basis as they suffered from cognitive impairment due to traumatic brain injury resulting from MVA or an organic mental disorder. Further four were removed based on their low accuracy (details are 
provided in Data Analysis section below). The division into PTSD and non-PTSD control group was based on the results of the psychiatric diagnosis following criteria defined in DSM-IV-TR (American Psychiatric Association, 2000). Importantly, the two groups did not differ regarding their age, sex distribution, the proportion of participants who completed higher education, as well as the time that elapsed since the MVA (all details reported in Table 1). Informed consent had been obtained from all the participants prior to testing.

\section{Measures}

\section{Psychopathology}

To establish the pre-treatment diagnoses entire Structured Clinical Interview for DSM-IV Axis I Disorders (SCID-I P/R; First et al., 1995) was administered to all the participants. PTSD was assessed using the SCID-I P/R Module F component (2008 revision). The SCID-I interviews were performed by five experienced psychiatrists. They were audio-recorded and 30\% of them were rated by a different assessor for reliability, showing the good strength of agreement $\left(\kappa=0.70^{*}, 84,5 \%\right.$ concordance of diagnoses). All these tools were adapted to the Polish language and have good psychometric properties (Zawadzki et al., 2015). Sample characteristics of both groups (Table 1) also include information about symptoms operationalized as their sum gathered in criteria B, C, and D during the interview establishing the PTSD diagnosis.

\section{The Pictorial EST}

In our choice of the EST characteristics, we aimed at maximizing the probability of evoking ESE while controlling for the potential confounding factors, which was of particular relevance given the socio-demographic heterogeneity of the sample. Drawing on the results of metaanalysis by Phaf and Kan (2007), we decided to present the stimuli in blocks and optimally, i.e., without any attempts to limit their conscious perception. The homogeneity of the stressor affecting our sample motivated the use of pictorial material that was relevant to the experiences of all the participants and, unlike words, should be processed similarly regardless of the level of education or reading proficiency.

Four categories of stimuli were used in the pictorial EST: positive, negative, neutral, and accident photos. All the photos were selected from the International Affective Picture system (IAPS; Lang et al., 1999), except accident photos, which were made available by Archives of Warsaw Police (11 photos) and supplemented by a single car accident photo from IAPS. All the accident photos had to depict crashed cars and accident scenes, but with no visible corps, injured bodies or blood, as we wanted the emotional interference to be specifically related to the memories of the accident, and not evoked by more general negative reactions (e.g., the view of mutilated bodies). Detailed list of the selected pictures from IAPS and their ratings might be found in Appendix A.

Each trial started with a black fixation cross $(1000 \mathrm{~ms})$, than a photo appeared for $600 \mathrm{~ms}$, followed by a colored rectangle fully masking the picture and remaining on the screen until a response was made or $3000 \mathrm{~ms}$ elapsed. The stimuli were presented on a 15-inch color monitor using E-prime software (version 1.1.4.1; Schneider et al., 2002). Participants were asked to look at the screen and name the color (red, yellow, green, or blue) of the rectangle appearing right after the photo was presented. Responses were given by pressing one of four keys $(\mathrm{z}, \mathrm{x}, \mathrm{n}$, and $\mathrm{m}$ on a standard keyboard). The instruction underlined that both speed and accuracy of reactions were of equal importance. As we expected older participants to show a general slowing of the reactions, the pictures and color patches masking them were presented sequentially so that all participants were exposed to the stimuli for the exact same amount of time, independent of their response speed. After 12 practice trials, participants solved four blocks of trials. In every block all photos from a single stimulus category were presented, each appeared four times coupled once with each of the colors, forming 48 trials per block. At the end of each block participants could take a short break, before pressing the spacebar to start the next set of trials. Blocks and trial order was fully randomized across participants.

TABLE 1 | Sample characteristics.

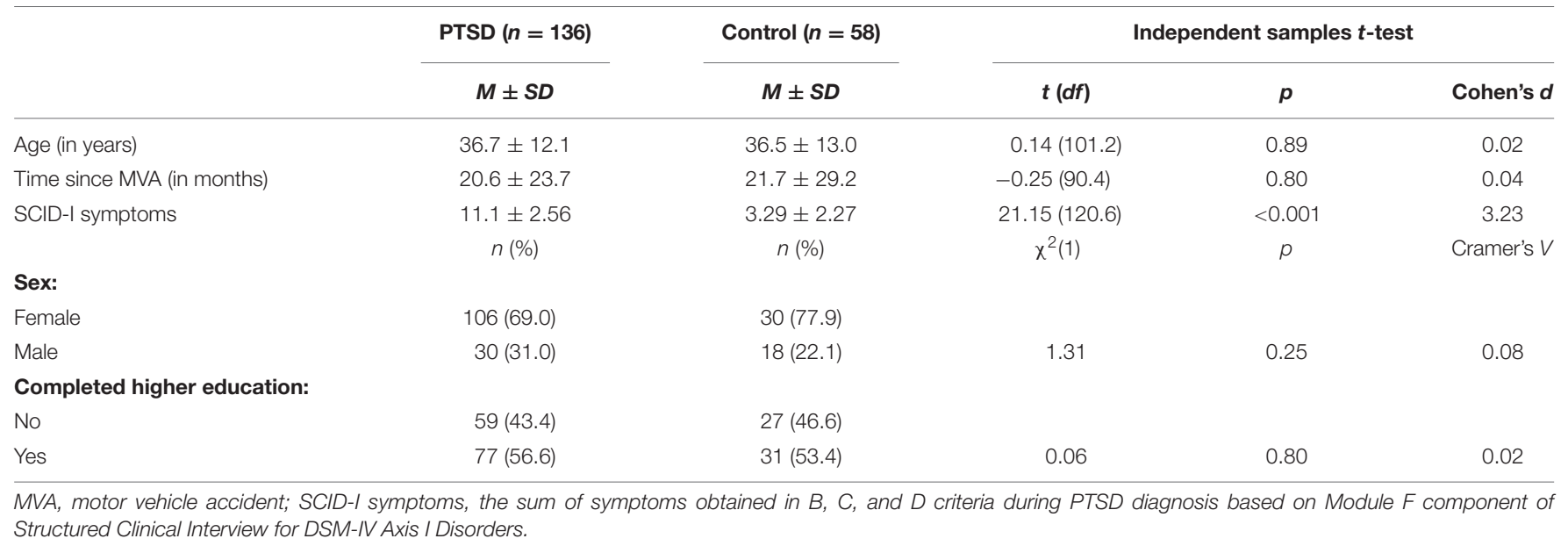




\section{Procedure}

All participants were tested individually. The pictorial EST was solved as the last part of a larger battery of computerized tests. Notably, none of the tasks preceding the EST employed pictorial material related to MVAs. Testing session lasted approximately $50 \mathrm{~min}$ and was performed shortly before the diagnostic interview.

\section{Data Analysis}

Results from four participants with distinctively low accuracy levels in the pictorial EST were removed prior to the analysis (in removed cases mean accuracy in at least one of the conditions was below 0.5 threshold, for the cases remaining in the analysis all accuracies were above 0.70). Demographic and basic clinical characteristics of the remaining 194 subjects are reported in Table 1. Analyses were conducted using three groups of indicators: (a) accuracy means, (b) RT means, and (c) difference scores computed by subtracting mean of RTs in neutral trials from mean scores computed for each of the remaining conditions (positive, negative, and accident photos). All incorrect responses and first trial in each block were removed from RT analysis. RTs shorter than $300 \mathrm{~ms}$ and longer than 3 SDs above the subject's block mean were also discarded (RT trimming removed $0.79 \%$ of all observations). In the first step of our analysis, we focused on the influence of PTSD diagnosis on all the dependent variables. In the second step - on the moderating effects of age.

\section{RESULTS}

\section{Effects Related to PTSD Diagnosis}

\section{Accuracy}

Mixed-design ANOVA was used to compare mean accuracies with Diagnosis (PTSD group vs. no PTSD) as between-subject factor and Photo Type (Neutral, Positive, Negative, Accident) as the within-subject variable. Results revealed a significant main effect of the Photo Type, $F(2.1,398.1)=11.79, p<0.001$, $\eta_{\mathrm{p}}^{2}=0.06$. Due to the violation of the sphericity assumption effects including Photo Type in all analyses are reported with Greenhouse-Geisser correction). Tukey post hoc tests indicated that performance in Accident condition $(M=96.3 \%$, $S D=5.59 \%)$ was significantly impaired when compared with Positive $(M=98.2 \%, S D=2.37 \%, p<0.001)$ and Neutral $(M=98.0 \%, S D=3.00 \%, p<0.001)$ trials and did not differ significantly when contrasted with trials presenting Negative material $(M=97.0 \%, S D=4.36 \%, p=0.133)$. Negative trials yielded lower accuracies when compared to trials with Positive pictures $(p=0.009)$. The remaining post hoc comparisons were non-significant. No effects related to the PTSD diagnosis were present, as both the main effect and the Diagnosis $\times$ Photo Type interaction were non-significant $(F \mathrm{~s}<1)$.

\section{RT Mean Scores}

In RT analysis we used the same ANOVA design as previously. As expected, the main effect of Photo Type $[F(2.6,495.9)=45.05$, $\left.p<0.001, \eta_{\mathrm{p}}^{2}=0.19\right]$ was accompanied by the two-way interaction with Diagnosis, $F(2.6,495.9)=3.08, p=0.034$,

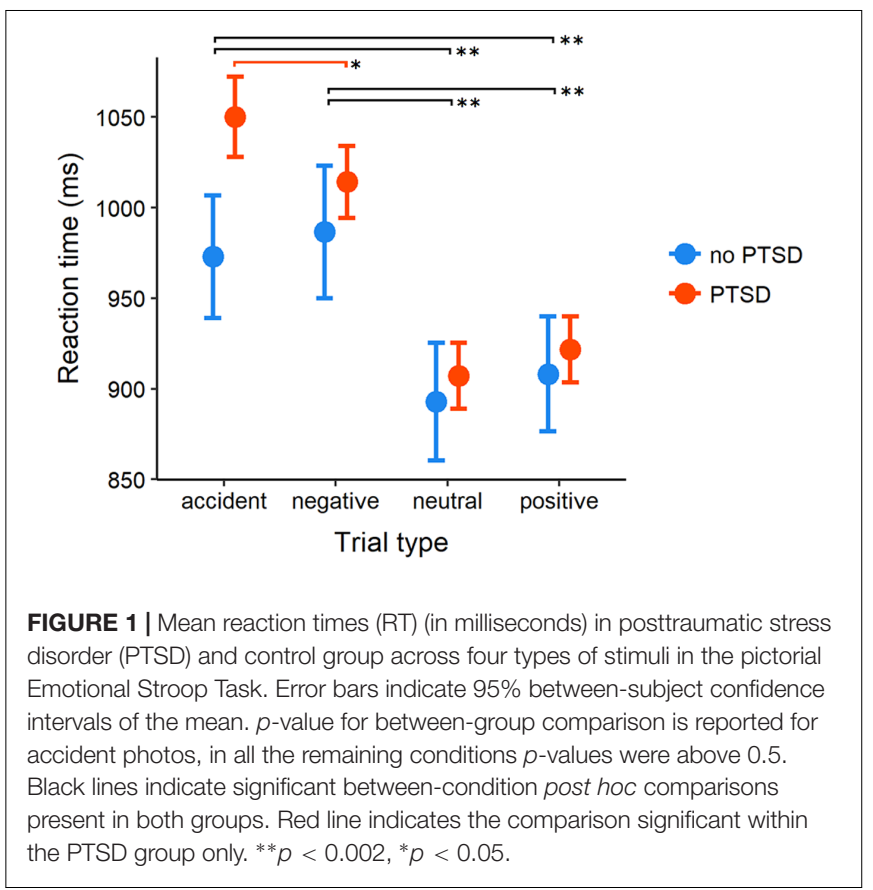

$\eta_{\mathrm{p}}^{2}=0.02$ (Figure 1). The main effect of Diagnosis was non-significant $(F<1)$. To disentangle the interaction effect, we performed analyses within groups. The main effect of Photo Type was present both in PTSD $[F(2.4,327.5)=50.66, p<0.001$, $\left.\eta_{\mathrm{p}}^{2}=0.27\right]$ and no PTSD participants $[F(2.8,158.7)=13.83$, $\left.p<0.001, \eta_{\mathrm{p}}^{2}=0.20\right]$, however, the pattern of results was markedly different. In both groups, Tukey's post hoc tests showed that RTs in neutral and positive trials were shorter than in conditions were accident and negative photos were presented (all ps $\leq 0.002$ ). Direct comparison of accident and negative trials showed, however, that significant increase in RTs related to the presentation of trauma-related material characterized only participants with $\operatorname{PTSD}(p=0.049, d=0.23)$ with no equivalent result in the control group $(p=0.867, d=-0.09$ ). Between-group comparison of RTs in Accident trials did not reach statistical significance, $t(107.91)=1.91, p=0.059, d=0.30$. (This and all the following independent samples $t$-test results are reported with Welch correction). Between subject comparisons in the remaining three types of trials were also non-significant (ps $>0.5,|d| \leq 0.11)$.

\section{RT Difference Scores}

Analysis of difference scores was based on $2 \times 3$ mixeddesign ANOVA with two levels of Diagnosis and three levels of Photo Type (Positive, Negative, and Accident conditions, Figure 2). Results revealed that the main effects of Photo Type $\left[F(1.9,369.2)=35.81, p<0.001, \eta_{p}^{2}=0.16\right]$ was accompanied by a significant interaction, $F(1.9,369.2)=3.58, p=0.031$, $\eta_{\mathrm{p}}^{2}=0.02$. The main effect of Diagnosis was not significant, $\left.F(1,192)=1.85, p=0.175, \eta_{\mathrm{p}}^{2}=0.01\right]$. Separate analyses in both groups showed that the Photo Type influenced interference scores in both groups, however, the effect in the PTSD group was more pronounced $\left[F(1.84,247.7)=43.50, p<0.001, \eta_{\mathrm{p}}^{2}=0.24\right]$ 


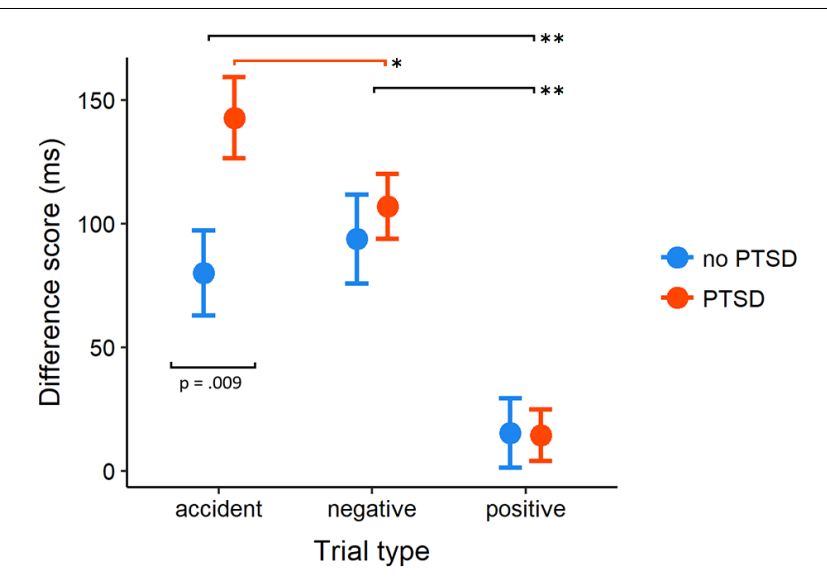

FIGURE 2 | Mean interference score of RTs (in milliseconds) in PTSD and control group computed as the difference between mean RT in negative, positive, and accident vs. neutral trials in the pictorial emotional Stroop task. Error bars indicate 95\% between-subject confidence intervals of the mean. $p$-value for between-group comparison is reported for accident photos, in all remaining conditions $p$-values were above 0.5 . Black lines indicate significant between-condition post hoc comparisons present in both groups. Red line indicates the comparison significant within the PTSD group only. ${ }^{* *} p<0.002$, ${ }^{*} p<0.05$.

than in non-PTSD subjects, $F(1.96,112.0)=9.95, p<0.001$, $\eta_{\mathrm{p}}^{2}=0.15$. The pattern of within-group pairwise comparisons matched exactly the one observed in the previous analysis for the Positive, Negative, and Accident conditions (for each subject the same value was subtracted to compute the interference scores, so relative results did not change). The difference scores allowed to reveal a significant between-group effect. When subjects diagnosed with PTSD were contrasted with control participants, their average scores in Accident trials were significantly higher $[t(164.5)=2.64, p=0.009, d=0.35]$, whereas virtually no effects were present in the interference scores in negative and positive trials $(p s>0.5,|d| \leq 0.09)$.

\section{Moderating Effects of Ages}

Regression analysis was conducted to address the crucial question of the moderating role of age on the relationship between the PTSD diagnosis and ESE. Separate regression models were fitted for two key indicators: mean RTs in which trauma-related photos were presented and interference scores obtained for the same trials. Both models were estimated using HC3 heteroskedasticity-consistent standard error estimators (sandwich package implementation, Zeileis, 2006) using $\mathrm{R}$ statistical environment ( $\mathrm{R}$ Core Team, 2016). Both analyses revealed the significant interaction of Age and Diagnosis on ESE (summaries of both models are presented in Table 2). To elucidate the moderating effects of age we used the Johnson-Neyman procedure (Hayes and Matthes, 2009). This technique allows to estimate the size of the effect related to the independent variable over the full spectrum of moderator values. Using Johnson-Neyman approach, we determined the "regions of significance," i.e., age ranges for which the PTSD diagnosis modifies significantly the performance. Figure $\mathbf{3 A}$ shows that estimated value of the conditional influence of PTSD diagnosis on mean RTs decreased systematically becoming non-significant for participants above the age of 36.8 and even significantly negative for participants above the age of 66.6. This latter value is close to the upper range of the age values observed in our sample, as the oldest tested participant was 69. Similar results could be observed for difference scores (Figure 3B), however, in this analysis region of significance reached up to the value of 39.7. It is important to underline that obtained cutoff scores are determined not only by the slope of the conditional effect but also by the breadth of the confidence intervals. Estimated conditional effects of PTSD diagnosis reached zero (points where the conditional effect crosses the horizontal axis) at higher age values: 45.3 and 51.7 years of age.

Potentially, the effects of age could also be confounded by not taking into account the time elapsed between the MVA and the diagnosis. That was, however, not the case in our study. The amount of time that passed since the accident was not related to any of the variables in models reported above there were no group differences (see Table 1) and correlations with age as well as both RT measures were non-significant and negligible (all $|r h o|<0.1$ and $p \geq 0.2$ ). Same correlations were also estimated separately within PTSD and control group, again not yielding any significant results. There was also no significant correlation between participants' age and the number of symptoms, as measured by SCID-I.

Additionally, we checked if the moderating effects reported above remained significant after adding participant sex or level of education as covariates. As none of the additional effects were significant, detailed descriptions of the results will be skipped for the sake of brevity.

\section{DISCUSSION}

According to our knowledge, this is one of the few studies focusing on the influence of age on ESE and the first one examining such a large sample of trauma victims. Using regression approach we showed that the interference effect observed in reaction to trauma-related photos in PTSD patients systematically decreased with subject's age. Importantly, moderating effects of age were present both in RTs and interference measures and could not be explained by differences in other patient characteristics (sex, the level of education, time since MVA).

It is worth to notice that the lack of ESE in the older group of subjects diagnosed with PTSD is coherent with two different lines of interpretation. It might either be related to the diminished sensitivity to accident photos in older subjects with PTSD or relative increase in the emotional interference in the control group. Given the scant literature on the aging effects on ESE, deciding between these two interpretations is a non-trivial task.

First, we focus on the explanations supporting the hypothesis of reduced interference in the PTSD group. The mechanism responsible for diminished ESE could be a specific case of a broader phenomenon observed in older healthy subjects. In the general population, older people are characterized by a 
TABLE 2 | Summary of regression models describing the association between PTSD diagnosis and pictorial emotional Stroop task performance including age as a moderating variable.

\begin{tabular}{|c|c|c|c|c|c|c|c|c|}
\hline & \multicolumn{4}{|c|}{ DV: Mean reactions times } & \multicolumn{4}{|c|}{ DV: Difference scores } \\
\hline & $B$ & $S E$ & $t$ & $p$ & $B$ & $S E$ & $t$ & $p$ \\
\hline Intercept & 615.5 & 84.5 & & & 97.2 & 52.1 & & \\
\hline Age & 9.8 & 2.2 & 4.56 & $<0.001$ & -0.5 & 1.5 & -0.31 & 0.756 \\
\hline Diagnosis & 393.3 & 106.4 & 3.70 & $<0.001$ & 216.4 & 67.1 & 3.23 & 0.001 \\
\hline Age $\times$ Diagnosis & -8.7 & 2.8 & -3.10 & 0.002 & -4.2 & 1.9 & -2.25 & 0.025 \\
\hline
\end{tabular}

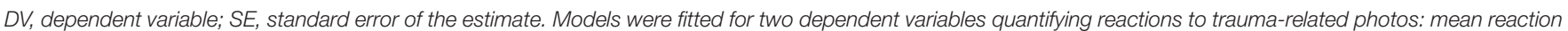


PTSD group = 1).


FIGURE 3 | Johnson-Neyman regions of significance for the conditional effect of Diagnosis on mean RT in accident photo trials (A) and mean interference scores (computed as the difference between accident and neutral photos conditions, B) in pictorial emotional Stroop task. Color bands depict 95\% confidence intervals blue regions indicate significant and red - non-significant conditional effects of Diagnosis at given age.

more effective emotional regulation (Scheibe and Carstensen, 2010; Hay and Diehl, 2011) and their attentional processes are biased toward positive stimuli (Reed et al., 2014). Furthermore, many studies indicate that in this group some components of the physiological reactions evoked by negative material are markedly different. Such effects were reported in studies using cardiovascular (e.g., lower heart rate reactivity, Uchino et al., 2010), breathing (e.g., no effects of arousal on inspiratory time, Gomez et al., 2016) and neuroimaging data (e.g., investigating the hypothesis of fronto-amygdalar age-related differences in emotion, St. Jacques et al., 2009). Potentially, age-related dampening of various components of physiological reactions (either peripheral or central) evoked by emotional material could also help explain the reduction of interference observed in older PTSD patients.

However, even if we ignore all the contradicting results and moderators of the effects listed above (e.g., Wurm et al., 2004; Uchino et al., 2010), it is important to notice that these explanations point toward more successful regulatory processes allowing older subjects to achieve positive emotional balance. That optimistic picture, described in the title of a review paper by Mather (2012) as "The emotion paradox in the aging brain," does not seem to be adequate when juxtaposed with the core symptoms of PTSD (Shepherd and Wild, 2014). Intense anxiety, reexperiencing, and intrusive thoughts are the exact opposite of superior emotional control. Attributing more effective regulation to the older PTSD group is even less convincing, in the light of recent neuroimaging data. Effective coping with trauma-related
TABLE 3 | List of photos from the International Affective Picture System used as stimuli in pictorial version of emotional Stroop task.

\begin{tabular}{cccc}
\hline & \multicolumn{3}{c}{ Identification numbers of selected photos } \\
\cline { 2 - 4 } & Negative & Neutral & Positive \\
\hline 2800 & 1670 & 1440 \\
2900 & 2200 & 1710 \\
3230 & 2215 & 1750 \\
& 3300 & 2372 & 1920 \\
6260 & 2749 & 2040 \\
& 6313 & 5534 & 2150 \\
& 6350 & 7002 & 2165 \\
& 9000 & 7009 & 2340 \\
9001 & 7035 & 2540 \\
& 9040 & 7050 & 2550 \\
& 9041 & 7150 & 5760 \\
Valence ratings & 9570 & 7705 & 7330 \\
\hline Arousal ratings & $2.27(0.50)$ & $5.07(0.59)$ & $7.97(0.25)$ \\
\hline Ratig & $5.50(1.17)$ & $3.12(0.45)$ & $4.54(0.59)$ \\
\hline
\end{tabular}

Ratings and numbering based on Lang et al. (1999).

stimuli correlates with increased top-down attentional control (White et al., 2015), whereas the behavioral data show that PTSD patients are characterized by lower levels of executive control both before and after the occurrence the traumatic stressor (Buckley, 2000; Aupperle et al., 2012). In sum, in our opinion, the 
age-related improvement in emotional regulation cannot account for the pattern of results observed in our study.

A more convincing explanation of the reduced ESE refers to the situational factors which are known to moderate the size of emotional interference and diversely influence participants of different ages. ESE is diminished if participants expect a confrontation with a significant real environmental stressor (Helfinstein et al., 2008). Similar effects were also observed in PTSD (Constans et al., 2004). Concerning the fact that the age range in our sample exceeded 50 years, it is likely that cohort effects and natural correlates of the aging might have shaped subjects reactions to the experimental task. In particular, both computerized testing situation and prospects of getting a psychiatric evaluation might have been perceived as stronger stressors by older subjects and, in consequence, affect the ESE in our study. Regrettably, since no measures of stress or emotional state were used during this part of the diagnostic process, we could not empirically verify this hypothesis. Future studies would certainly benefit from including momentary measurements of mood and stress levels as additional controlled variables.

The second line of reasoning explaining observed results refers to the hypothesis that aging makes all study participants including the control group - more susceptible to the emotional load imposed by accident photos. It is important to underline that our sample consisted of people who decided to seek help and undergo a psychiatric evaluation following an MVA (for a detailed description of the treatment program see: Popiel et al., 2015). Hence, for every subject in our study accident pictures related to a significant, emotionally charged personal experience. Older adults are characterized not only by a general depletion of cognitive resources (e.g., Verhaeghen and Salthouse, 1997), but also by more specific changes in their emotional reactions including a gradual increase in aversive activation and the strength of arousal evoked by displeasure (Keil and Freund, 2009). The modulating effects of age on cognition-emotion interaction also translate into systematic changes in the perception of emotionally charged stimuli. As shown by Grühn and Scheibe (2008) older participants' ratings of negative pictures from IAPS database are more extreme in terms of both affect and arousal. As mentioned before, in many contexts older people outperform younger groups in tasks requiring emotional regulation. This advantage, however, is not ubiquitous and several studies indicate that effects of superior emotional control might dissipate or even be reversed in certain conditions. For example, the positivity effects seem to rely on the cognitive-control-based allocation of attention. Therefore, the tendency to avoid negative stimuli is reversed if control processes are disrupted by the cognitive load imposed by a secondary task (Knight et al., 2007). Results obtained by Tomaszczyk et al. (2008) suggest that the positivity bias in memory recall might be also significantly reduced if participants are presented with pictures of high personal relevance (as it was the case in our study).

This line of reasoning might seem incompatible with the existing data concerning the ESE in elderly subjects'. It is known that ESE is preserved in older age in many groups including, for example, subjects who are depressed (Dudley et al., 2002; Epp et al., 2012) or habitually worrying (Price et al., 2012). Older participants are generally slower, but the emotional interference effects might be reliably observed. We should keep in mind, however, that these results are obtained in research involving healthy controls, whereas in our study, all participants were affected by a significant stressor - MVA. Even though the intensity of symptoms was much higher in PTSD, the control subjects were not fully asymptomatic (see: Table 1., SCIDI symptoms' measure). At the same time, current knowledge about the ESE phenomenon (Phaf and Kan, 2007) suggests that it does not rely on the same regulatory mechanisms as the ones which are known to be more effective in older adults. For example, it would be hard to explain how ESE could be reduced by consciously changing the strategy of attention allocation (as it was the case in studies mentioned above).

All these results suggest that the older MVA victims, even if not presenting full spectrum of PTSD symptoms, might face more problems with effective functioning in accident trials when compared with younger subjects, and this could lead to the reduction of the between-group differences in ESE in our study. Given the complexity and variety of theoretical contexts, the in-depth analysis of the mediators responsible for diminished ESE in older participants remains as a task to be explored. The list of candidate mechanisms outlined above is certainly non-exhaustive, and it is also theoretically plausible that some of them might work in parallel. Moreover, our study design did not allow us to systematically investigate the time dynamics of the ESE, which is a topic of important theoretical implications (see e.g., Kunde and Mauer, 2008; BenHaim et al., 2014). In our procedure, each of the photos was presented four times in a fully randomized manner, hence changes in the ESE due to the stimulus repetition could not be disentangled from other important factors (gradual mood changes, habituation, etc.). More in-depth analysis of this aspect of participants' performance is a task of the future.

Due to its exploratory character, our study certainly requires further validation. If such efforts were successful, the conclusions would bear important implications for the understanding of ESE from a measurement-oriented perspective. Tasks used in cognitive psychopathology research are often perceived as if their validity across socio-demographic dimensions were warranted. Our study might be treated as a reminder, that psychometric properties of any tool should be re-established whenever it is used beyond the typically tested population. That conclusion becomes even more relevant given the rapid aging of modern societies (United Nations, 2015) and the breadth of ESE usage, including studies on the effectiveness of therapeutic interventions (e.g., El Khoury-Malhame Myriam et al., 2011), neural underpinnings of various disorders (e.g., Herd et al., 2006; Cisler et al., 2011; Shvil et al., 2013) or relapse prediction in addicts (e.g., Waters et al., 2003; Streeter et al., 2008).

To summarize, our results point toward the importance of age as a variable moderating the presence of ESE in PTSD patients 
when contrasted with trauma-exposed controls. Concerning the exploratory nature of the study, reported results certainly require independent replication also allowing to determine to what extent our findings generalize to other clinical populations and different versions of EST, in particular - ones based on non-pictorial stimuli. It is important to underline, however, that current knowledge about the determinants of ESE, the emergence of the expected interference effects in younger subjects, and exceptionally large clinical sample add credibility to the postulated age-related decline of ESE.

\section{ETHICS STATEMENT}

This study was carried out in accordance with the recommendations of the institutional ethics committees at the University of Warsaw and the Military Institute of Aviation Medicine in Warsaw. All subjects gave written informed consent in accordance with the Declaration of Helsinki. The protocol was approved by the institutional ethics committees at the University of Warsaw and the Military Institute of Aviation Medicine in Warsaw.

\section{REFERENCES}

American Psychiatric Association (2000). Diagnostic and Statistical Manual of Mental Disorders, 4th Edn. Washington, DC: Author.

Ashley, V., and Swick, D. (2009). Consequences of emotional stimuli: age differences on pure and mixed blocks of the emotional Stroop. Behav. Brain Funct. 5:14. doi: 10.1186/1744-9081-5-14

Aupperle, R. L., Melrose, A. J., Stein, M. B., and Paulus, M. P. (2012). Executive function and PTSD: disengaging from trauma. Neuropharmacology 62, 686-694. doi: 10.1016/j.neuropharm.2011.02.008

Ben-David, B. M., Chajut, E., and Algom, D. (2012). The pale shades of emotion: a signal detection theory analysis of the emotional Stroop task. Psychology 3, 537-541. doi: 10.4236/psych.2012.37079

Ben-Haim, M. S., Mama, Y., Icht, M., and Algom, D. (2014). Is the emotional Stroop task a special case of mood induction? Evidence from sustained effects of attention under emotion. Attent. Percept. Psychophys. 76, 81-97. doi: 10.3758/ s13414-013-0545-7

Bruce, S. E., Buchholz, K. R., Brown, W. J., Yan, L., Durbin, A., and Sheline, Y. I. (2013). Altered emotional interference processing in the amygdala and insula in women with post-traumatic stress disorder. Neuroimage Clin. 2, 43-49. doi: 10.1016/j.nicl.2012.11.003

Buckley, T. (2000). Information processing and PTSD: a review of the empirical literature. Clin. Psychol. Rev. 20, 1041-1065. doi: 10.1016/S0272-7358(99) 00030-6

Cisler, J. M., and Koster, E. H. W. (2010). Mechanisms of attentional biases towards threat in anxiety disorders: an integrative review. Clin. Psychol. Rev. 30, 203-216. doi: 10.1016/j.cpr.2009.11.003

Cisler, J. M., Wolitzky-Taylor, K. B., Adams, T. G., Babson, K. A., Badour, C. L., and Willems, J. L. (2011). The emotional Stroop task and posttraumatic stress disorder: a meta-analysis. Clin. Psychol. Rev. 31, 817-828. doi: 10.1016/j.cpr. 2011.03.007

Constans, J. I., McCloskey, M. S., Vasterling, J. J., Brailey, K., and Mathews, A. (2004). Suppression of attentional bias in PTSD. J. Abnorm. Psychol. 113, 315-323. doi: 10.1037/0021-843X.113.2.315

Devineni, T., Blanchard, E. B., Hickling, E. J., and Buckley, T. C. (2004). Effect of psychological treatment on cognitive bias in motor vehicle accident-related posttraumatic stress disorder. J. Anxiety Disord. 18, 211-231. doi: 10.1016/ S0887-6185(02)00247-5

Dudley, R., O’Brien, J., Barnett, N., McGuckin, L., and Britton, P. (2002). Distinguishing depression from dementia in later life: a pilot study employing

\section{AUTHOR CONTRIBUTIONS}

All the authors had contribution to the conception of the work and participated in the writing of the manuscript. MB was the lead author and primary writer. MB and GS contributed to the experimental procedure design. Data collection and psychiatric diagnoses were performed under the supervision of AP and BZ. MB performed data analysis. All authors approved the final version of the manuscript.

\section{FUNDING}

This paper was supported by Grant 2012/06/A/HS6/00340 "PTSD: Diagnosis Therapy Prevention" from National Science Centre, Poland. The data were collected during the TRAKT study supported by the Financial Mechanism Committee established by Iceland, Liechtenstein, and Norway through EEA Mechanism and the Polish Ministry of Science and Higher Education, Grant PL0088 "Psychological Causes and Consequences of Traffic Accidents" awarded to BZ at the University of Warsaw.

the Emotional Stroop task. Int. J. Geriatr. Psychiatry 17, 48-53. doi: 10.1002/ gps. 514

El Khoury-Malhame Myriam, M., Lanteaume, L., Beetz, E. M., Roques, J., Reynaud, E., Samuelian, J. C., et al. (2011). Attentional bias in post-traumatic stress disorder diminishes after symptom amelioration. Behav. Res. Ther. 49, 796-801. doi: 10.1016/j.brat.2011.08.006

Epp, A. M., Dobson, K. S., Dozois, D. J. A., and Frewen, P. A. (2012). A systematic meta-analysis of the Stroop task in depression. Clin. Psychol. Rev. 32, 316-328. doi: 10.1016/j.cpr.2012.02.005

First, M., Spitzer, R., Gibbon, M., and Williams, J. (1995). Structured Clinical Interview for DSM-IV Axis I Disorders. New York, NY: New York State Psychiatric Institute.

Foa, E. B., and Rauch, S. A. M. (2004). Cognitive changes during prolonged exposure versus prolonged exposure plus cognitive restructuring in female assault survivors with posttraumatic stress disorder. J. Consult. Clin. Psychol. 72, 879-884. doi: 10.1037/0022-006X.72.5.879

Gomez, P., Filippou, D., Pais, B., von Gunten, A., and Danuser, B. (2016). Breathing and affective picture processing across the adult lifespan. Biol. Psychol. 119, 101-111. doi: 10.1016/j.biopsycho.2016.07.011

Gropalis, M., Bleichhardt, G., Hiller, W., and Witthöft, M. (2013). Specificity and modifiability of cognitive biases in hypochondriasis. J. Consult. Clin. Psychol. 81, 558-565. doi: 10.1037/a0028493

Grühn, D., and Scheibe, S. (2008). Age-related differences in valence and arousal ratings of pictures from the International Affective Picture System (IAPS): do ratings become more extreme with age? Behav. Res. Methods 40, 512-521. doi: 10.3758/BRM.40.2.512

Hay, E. L., and Diehl, M. (2011). Emotion complexity and emotion regulation across adulthood. Eur. J. Ageing 8, 157-168. doi: 10.1007/s10433-0110191-7

Hayes, A. F., and Matthes, J. (2009). Computational procedures for probing interactions in OLS and logistic regression: SPSS and SAS implementations. Behav. Res. Methods 41, 924-936. doi: 10.3758/BRM. 41.3.924

Helfinstein, S. M., White, L. K., Bar-Haim, Y., and Fox, N. A. (2008). Affective primes suppress attention bias to threat in socially anxious individuals. Behav. Res. Ther. 46, 799-810. doi: 10.1016/j.brat.2008. 03.011

Herd, S. A., Banich, M. T., and O'Reilly, R. C. (2006). Neural mechanisms of cognitive control: an integrative model of Stroop task performance and FMRI data. J. Cogn. Neurosci. 18, 22-32. doi: 10.1162/089892906775250012 
Keil, A., and Freund, A. M. (2009). Changes in the sensitivity to appetitive and aversive arousal across adulthood. Psychol. Aging 24, 668-680. doi: 10.1037/ a0016969

Khanna, M. M., Badura-Brack, A. S., McDermott, T. J., Shepherd, A., HeinrichsGraham, E., Pine, D. S., et al. (2016). Attention training normalises combatrelated post-traumatic stress disorder effects on emotional Stroop performance using lexically matched word lists. Cogn. Emot. 30, 1521-1528. doi: 10.1080/ 02699931.2015.1076769

Kimble, M. O., Frueh, B. C., and Marks, L. (2009). Does the modified Stroop effect exist in PTSD? Evidence from dissertation abstracts and the peer reviewed literature. J. Anxiety Disord. 23, 650-655. doi: 10.1016/j.janxdis.2009.02.002

Knight, M., Seymour, T. L., Gaunt, J. T., Baker, C., Nesmith, K., and Mather, M. (2007). Aging and goal-directed emotional attention: distraction reverses emotional biases. Emotion 7, 705-714. doi: 10.1037/1528-3542.7.4.705

Kunde, W., and Mauer, N. (2008). Sequential modulations of valence processing in the emotional Stroop task. Exp. Psychol. 55, 151-156. doi: 10.1027/1618-3169. 55.3.151

Lamonica, H. M., Keefe, R. S. E., Harvey, P. D., Gold, J. M., and Goldberg, T. E. (2010). Differential effects of emotional information on interference task performance across the life span. Front. Aging Neurosci. 2:141. doi: 10.3389/ fnagi.2010.00141

Lang, P. J., Bradley, M. M., and Cuthbert, N. B. (1999). International Affective Picture System (IAPS): Instruction Manual and Affective Ratings. Technical report A-4. Gainesville, FL: University of Florida.

Mather, M. (2012). The emotion paradox in the aging brain. Ann. N. Y. Acad. Sci. 1251, 33-49. doi: 10.1111/j.1749-6632.2012.06471.x

McLeod, C. (2005). “The Stroop task in clinical research," in Cognitive Methods and their Application to Clinical Research, eds A. Wencel and D. C. Rubin (Washington, DC: American Psychological Association), 41-62.

Morgan, E. S., and Scheibe, S. (2013). "Reconciling cognitive decline and increased well-being with age," in The Oxford Handbook of Emotion, Social Cognition, and Problem Solving in Adulthood, eds P. Verhaeghen and C. Hertzog (New York, NY: Oxford University Press), 155-172.

Norris, F. H., Kaniasty, K., Conrad, M. L., Inman, G. L., and Murphy, A. D. (2002). Placing age differences in cultural context : a comparison of the effects of age on PTSD after disasters in the United States, Mexico, and Poland. J. Clin. Geropsychol. 8, 153-173. doi: 10.1023/A:1015940126474

Phaf, R. H., and Kan, K. J. (2007). The automaticity of emotional Stroop: a metaanalysis. J. Behav. Ther. Exp. Psychiatry 38, 184-199. doi: 10.1016/j.jbtep.2006. 10.008

Popiel, A., Zawadzki, B., Pragłowska, E., and Teichman, Y. (2015). Prolonged exposure, paroxetine and the combination in the treatment of PTSD following a motor vehicle accident. A randomized clinical trial - The "TRAKT" study. J. Behav. Ther. Exp. Psychiatry 48, 17-26. doi: 10.1016/j.jbtep.2015.01.002

Price, R. B., Siegle, G., and Mohlman, J. (2012). Emotional Stroop performance in older adults. Am. J. Geriatr. Psychiatry 20, 798-805. doi: 10.1097/JGP. ob013e318230340d

R Core Team (2016). R: A Language and Environment for Statistical Computing. Vienna: The R Foundation for Statistical Computing.

Reed, A. E., Chan, L., and Mikels, J. A. (2014). Meta-analysis of the age-related positivity effect: age differences in preferences for positive over negative information. Psychol. Aging 29, 1-15. doi: 10.1037/a0035194

Scheibe, S., and Carstensen, L. L. (2010). Emotional aging: recent findings and future trends. J. Gerontol. Psychol. Sci. 65B, 135-144. doi: 10.1093/geronb/ gbp 132

Schneider, W., Eschman, A., and Zuccolotto, A. (2002). E-Prime User's Guide. Pittsburgh, PA: Psychology Software Tools Inc.

Shepherd, L., and Wild, J. (2014). Emotion regulation, physiological arousal and PTSD symptoms in trauma-exposed individuals. J. Behav. Ther. Exp. Psychiatry 45, 360-367. doi: 10.1016/j.jbtep.2014.03.002

Shvil, E., Rusch, H. L., Sullivan, G. M., and Neria, Y. (2013). Neural, psychophysiological, and behavioral markers of fear processing in PTSD: a review of the literature. Curr. Psychiatry Rep. 15:358. doi: 10.1007/s11920-0130358-3

St. Jacques, P. L., Bessette-Symons, B., and Cabeza, R. (2009). Functional neuroimaging studies of aging and emotion: fronto-amygdalar differences during emotional perception and episodic memory. J. Int. Neuropsychol. Soc. 15, 819-825. doi: 10.1017/S1355617709990439

Streeter, C. C., Terhune, D. B., Whitfield, T. H., Gruber, S., Sarid-Segal, O., Silveri, M. M., et al. (2008). Performance on the Stroop predicts treatment compliance in cocaine-dependent individuals. Neuropsychopharmacology 33, 827-836. doi: 10.1038/sj.npp.1301465

Tomaszczyk, J. C., Fernandes, M. A., and MacLeod, C. M. (2008). Personal relevance modulates the positivity bias in recall of emotional pictures in older adults. Psychon. Bull. Rev. 15, 191-196. doi: 10.3758/PBR. 15.1.191

Uchino, B. N., Birmingham, W., and Berg, C. A. (2010). Are older adults less or more physiologically reactive? A meta-analysis of age-related differences in cardiovascular reactivity to laboratory tasks. J. Gerontol. Ser. B Psychol. Sci. Soc. Sci. 65B, 154-162. doi: 10.1093/geronb/gbp127

United Nations (2015). World Population Prospects the 2015 Revision: Comprehensive Tables (ST/ESA/SER.A/379). Available at: https://esa.un. org/unpd/wpp/Publications/Files/WPP2015_Volume-I_ComprehensiveTables.pdf

Verhaeghen, P., and Salthouse, T. A. (1997). Meta-analyses of age-cognition relations in adulthood: estimates of linear and nonlinear age effects and structural models. Psychol. Bull. 122, 231-249. doi: 10.1037/0033-2909.122. 3.231

Waters, A. J., Shiffman, S., Sayette, M. A., Paty, J. A., Gwaltney, C. J., and Balabanis, M. H. (2003). Attentional bias predicts outcome in smoking cessation. Health Psychol. 22, 378-387. doi: 10.1037/0278-6133.22.4.378

White, S. F., Costanzo, M. E., Blair, J. R., and Roy, M. J. (2015). PTSD symptom severity is associated with increased recruitment of top-down attentional control in a trauma-exposed sample. Neuroimage Clin. 7, 19-27. doi: 10.1016/j. nicl.2014.11.012

Williams, J. M. G., Mathews, A., and MacLeod, C. (1996). The emotional Stroop task and psychopathology. Psychol. Bull. 120, 3-24. doi: 10.1037/0033-2909. 120.1 .3

Wittekind, C. E., Jelinek, L., Kellner, M., Moritz, S., and Muhtz, C. (2010). Intergenerational transmission of biased information processing in posttraumatic stress disorder (PTSD) following displacement after World War II. J. Anxiety Disord. 24, 953-957. doi: 10.1016/j.janxdis.2010. 06.023

Wittekind, C. E., Muhtz, C., Moritz, S., and Jelinek, L. (2017). Performance in a blocked versus randomized emotional Stroop task in an aged, early traumatized group with and without posttraumatic stress symptoms. J. Behav. Ther. Exp. Psychiatry 54, 35-43. doi: 10.1016/j.jbtep.2016.06.003

Wurm, L. H., Labouvie-Vief, G., Aycock, J., Rebucal, K. A., and Koch, H. E. (2004). Performance in auditory and visual emotional Stroop tasks: a comparison of older and younger adults. Psychol. Aging 19, 523-535. doi: 10.1037/0882-7974. 19.3.523

Xue, C., Ge, Y., Tang, B., Liu, Y., Kang, P., Wang, M., et al. (2015). A meta-analysis of risk factors for combat-related PTSD among military personnel and veterans. PLOS ONE 10:e0120270. doi: 10.1371/journal.pone. 0120270

Zawadzki, B., Popiel, A., Cyniak-Cieciura, M., Jakubowska, B., and Pragłowska, E. (2015). Diagnosis of posttraumatic stress disorder (PTSD) by the structured clinical interview SCID-I. Psychiatr. Pol. 49, 159-169. doi: 10.12740/PP/ 32214

Zeileis, A. (2006). Object-oriented computation of sandwich estimators. J. Stat. Softw. 16, 1-16. doi: 10.18637/jss.v016.i09

Conflict of Interest Statement: The authors declare that the research was conducted in the absence of any commercial or financial relationships that could be construed as a potential conflict of interest.

Copyright $\odot 2017$ Bielecki, Popiel, Zawadzki and Sedek. This is an open-access article distributed under the terms of the Creative Commons Attribution License (CC BY). The use, distribution or reproduction in other forums is permitted, provided the original author(s) or licensor are credited and that the original publication in this journal is cited, in accordance with accepted academic practice. No use, distribution or reproduction is permitted which does not comply with these terms. 


\section{APPENDIX A}

Three categories of photos used in the pictorial version of the emotional Stroop task (negative, neutral, and positive) were selected from International Affective Picture System (Lang et al., 1999). Table 3. provides a complete list of picture numbers and descriptive statistics summarizing affective properties of each category of stimuli. Valence, and arousal ratings and photos' numbers are based on Lang et al. (1999) publication (norms for both sexes). Selected pictures were horizontally oriented, did not depict any cars or elements that could be directly associated with accidents. In particular, negative pictures did not depict mutilated bodies or blood. Picture ratings are expressed on a 1 to 9 scale with 5 being the neutral middle-point of the valence scale. 